\title{
Targeted therapy for localized non-small-cell lung cancer: a review
}

This article was published in the following Dove Press journal:

OncoTargets and Therapy

5 July 2016

Number of times this article has been viewed

\author{
Nicolas Paleiron' \\ Olivier Bylicki² \\ Michel André \\ Emilie Rivière' \\ Frederic Grassin' \\ Gilles Robinet ${ }^{3}$ \\ Christos Chouaïd ${ }^{4}$ \\ On behalf of the GFPC \\ Group \\ 'Chest Department, HIA Clermont \\ Tonnerre, Brest, ${ }^{2}$ Chest Department, \\ HIA Percy, Clamart, ${ }^{3}$ Chest \\ Department, $\mathrm{CHU}$ de Brest, Brest, \\ ${ }^{4}$ GRC OncoEst, Université Paris XII, \\ Paris, France
}

\begin{abstract}
Targeted therapies have markedly improved the management of patients with advanced non-small-cell lung cancer (NSCLC), but their efficacy in localized NSCLC is less well established. The aim of this review is to analyze trials of targeted therapies in localized NSCLC. In patients with wild-type EGFR, tyrosine kinase inhibitors have shown no efficacy in Phase III trials. Few data are available for EGFR-mutated localized NSCLC, as routine biological profiling is not recommended. Available studies are small, often retrospectives, and/or conducted in a single-center making it difficult to draw firm conclusions. Ongoing prospective Phase III trials are comparing adjuvant tyrosine kinase inhibitor administration versus adjuvant chemotherapy. By analogy with the indication of bevacizumab in advanced NSCLC, use of antiangiogenic agents in the perioperative setting is currently restricted to nonsquamous NSCLC. Several trials of adjuvant or neoadjuvant bevacizumab are planned or ongoing, but for the moment there is no evidence of efficacy. Data on perioperative use of biomarkers in early-stage NSCLC come mainly from small, retrospective, uncontrolled studies. Assessment of customized adjuvant or neoadjuvant therapy in localized NSCLC (with or without oncogenic driver mutations) is a major challenge.
\end{abstract}

Keywords: targeted therapy, non-small-cell lung cancer, adjuvant, neo-adjuvant, surgery

\section{Introduction}

Tyrosine kinase inhibitors (TKIs) appear to have no place in adjuvant treatment of non-small-cell lung cancer (NSCLC) without a specific mutational profile. Prospective Phase III trials comparing adjuvant TKI with adjuvant chemotherapy in EGFR-mutated patients are ongoing. For the moment, there is no evidence that antiangiogenic agents are effective in the adjuvant setting. Customized adjuvant or neo/adjuvant therapy in localized NSCLC (with or without oncogenic driver mutations) remains a major challenge. Despite significant advances in the detection and management of lung cancer, it remains the leading cause of cancer-related mortality. Recent advances in the detection of genetic alterations, along with studies of physiologically relevant animal models, have yielded a new understanding of the molecular etiology of lung cancer. This has facilitated the development of potent and specific targeted therapies based on the genetic and biochemical alterations present in the tumor, especially NSCLC. It is now clear that heterogeneous cell signaling pathways are disrupted in NSCLC, including mutations in critical growth-regulating proteins (K-Ras, EGFR, B-RAF, MEK-1, HER2, MET, EML-4-ALK, KIF5B-RET, and NKX2.1) and inactivation of growth-inhibiting pathways (TP53, PTEN, p16, and LKB-1). Targeted therapies have markedly improved the management of patients with advanced NSCLC associated with $E G F R$ mutations or $E M L 4-A L K$ translocation, and also of patients 
with less frequent oncogenic mutations (BRAF or HER2 mutation, MET amplification, ROS1 or RET translocation). ${ }^{1,2}$ Targeted therapies have also proven effective in patients without a specific mutational profile. First-line combination therapy with bevacizumab, an antiangiogenic drug, plus platinum-based chemotherapy improves progression-free survival (PFS) and overall survival (OS) in patients with nonsquamous NSCLC. TKIs improve PFS when used as second- or third-line treatment, regardless of the tumor histology and mutational status. ${ }^{2}$

However, the efficacy of targeted therapies in localized NSCLC is less well established. The aim of this review is to analyze published studies of targeted therapies in localized NSCLC. Surgery is the optimal treatment for early-stage NSCLC. In patients undergoing surgery, a recent metaanalysis confirmed that adjuvant chemotherapy conferred a survival benefit of $4 \%-5 \%$ at 5 years in patients with stage II or III NSCLC. ${ }^{3}$ However, there was no benefit at stage IA, a questionable benefit at stage IB, and only a probable benefit in patients with tumors $>4 \mathrm{~cm} .{ }^{4-8}$ Similarly, an analysis of 15 randomized controlled trials showed that neoadjuvant chemotherapy conferred the same survival benefit (4\%-5\% at 5 years) as adjuvant chemotherapy in patients with stage IB-IIIA NSCLC. ${ }^{9}$

\section{TKIs in the perioperative setting Patients without a specific mutational profile}

Several trials have tested perioperative TKI therapy in patients without a specific mutational profile (Table 1). The BR19 study was intended to include 1,242 patients undergoing lobectomy or pneumonectomy for stage IB, II, or IIIA NSCLC. ${ }^{10}$ The patients were randomized, after stratification for age, sex, chemotherapy, or adjuvant radiotherapy, between gefitinib $250 \mathrm{mg}$ /day and placebo, given for 2 years. The primary endpoint was OS. The trial was halted prematurely after the inclusion of 503 patients, following the failure of first-line gefitinib in metastatic NSCLC, and it was therefore not possible to judge the value of gefitinib in this setting. The RADIANT trial included 973 patients undergoing surgical resection for stage IB to IIA NSCLC (with stratification for smoking, histology, tumor stage, adjuvant chemotherapy, and EGFR status by fluorescence in situ hybridization), and compared erlotinib with placebo. ${ }^{11}$ The trial showed no benefit of erlotinib in terms of PFS, the primary endpoint. Thus, adjuvant TKIs do not appear to be beneficial in NSCLC patients without a specific mutational profile.

\section{Patients with activating EGFR mutations}

Biological profiling is not recommended for patients with localized NSCLC, and few data on targeted therapies are therefore available in this population. ${ }^{12}$ A retrospective analysis of a randomized trial of adjuvant chemotherapy focused on 150 patients with IIIA-N2 NSCLC and known EGFR status. Regardless of adjuvant chemotherapy, the prognosis was better for $E G F R$-mutated patients (PFS, 41 vs 20 months; OS, 50 vs 25 months). Intriguingly, EGFR-wildtype patients who received adjuvant chemotherapy had longer PFS and OS (32 vs 14 months and 32 vs 17 months, respectively), while EGFR-mutated patients who received adjuvant chemotherapy had shorter PFS and OS (30 vs 49 and 33 vs 59 months, respectively). However, these results need to be confirmed in a prospective study. ${ }^{12}$

A single-center, nonrandomized trial included 167 patients with stage I/III, EGFR-mutated NSCLC. ${ }^{13}$ Multivariate analysis adjusted for sex, stage, type of surgery, and adjuvant chemotherapy showed a trend toward a higher 2-year survival rate in the 56 patients $(33 \%)$ who received a TKI perioperatively (96\% vs 90\%), but no firm conclusion can be drawn in the absence of randomization. ${ }^{13}$

An open, randomized, Phase II trial conducted in Asia, after surgery and adjuvant chemotherapy (four cycles of

Table I Trials in perioperative setting

\begin{tabular}{|c|c|c|c|}
\hline References & Design & Treatment tested & Results \\
\hline Goss et $\mathrm{al}^{10}$ & Phase III & Gefitinib versus placebo & Negative \\
\hline Altorki et al" & Phase III & Erlotinib versus placebo & Negative \\
\hline Sun et $\mathrm{al}^{12}$ & Retrospective & Chemotherapy & $\begin{array}{l}\text { Better outcome for EGFR wild- } \\
\text { type compared to EGFR mutation }\end{array}$ \\
\hline \multirow[t]{3}{*}{ Janjigian et $\mathrm{al}^{13}$} & Single-center & Tyrosine kinase inhibitor & Better outcome with tyrosine \\
\hline & No randomized & Chemotherapy & kinase inhibitor \\
\hline & EGFR-mutated patients & & \\
\hline Li et $\mathrm{al}^{14}$ & Phase II, randomized & $\begin{array}{l}\text { Tyrosine kinase inhibitor } \\
\text { versus chemotherapy }\end{array}$ & Negative \\
\hline
\end{tabular}

Abbreviation: EGFR, epidermal growth factor receptor. 
pemetrexed-carboplatin), compared a 6-month course of gefinib with simple monitoring in EGFR-mutated, stage IIIA-N2 NSCLC patients. PFS, the primary endpoint, was 39.8 months in the gefitinib arm and 27 months in the monitoring arm $(P=0.014)$ but only a trend toward better 2-year OS was observed (92.4\% vs $77.4 \%, P=0.07) .{ }^{14}$

The SELECT trial, a nonrandomized, prospective, multicenter study, included EGFR-mutated stage IA/IIIA NSCLC patients who received erlotinib $150 \mathrm{mg}$ /day for 2 years after surgery, plus adjuvant chemotherapy and/or adjuvant radiation therapy. ${ }^{15}$ Preliminary results for the first 100 patients (45\% in stage I, 27\% in stage II, and $28 \%$ in stage III) showed that $69 \%$ of patients received erlotinib for at least 22 months and that only $11 \%$ of them had to stop treatment during the first month. The primary endpoint, 2-year PFS, was $89 \%$, a rate higher than the "historical" value of 76\%. Two-year PFS was very high in stage I (96\%) and also in stage III (91\%). The main value of this trial is that it demonstrates the good feasibility of adjuvant treatment extended by TKI therapy in this population of EGFR-mutated patients. Biopsy of tumor recurrences showed the same mutation as that identified at initial diagnosis in the vast majority of cases. $7790 \mathrm{M}$, a mutation conferring resistance to first-generation TKIs, was found in rare cases (Table 1).

In the RADIANT study, 102 patients in the erlotinib arm and 59 patients in the placebo arm had EGFR-mutated tumors. The groups were similar in terms of sex, age, ethnicity, geographic origin, and smoking status, but the erlotinib arm included more stage IB/IIA disease (59\% vs $39 \%$ ) and less frequent adjuvant chemotherapy (45\% vs $56 \%) .{ }^{16}$ PFS was better in the erlotinib arm (46 vs 28 months, $P=0.04)$ but this result cannot really be considered statistically significant, as the conditions required for hierarchical analysis were not met. There was no difference in $\operatorname{OS}(P=0.8)$. For reasons that remain unclear, the proportion of patients with cerebral relapse was higher in the erlotinib arm (40\%) than in the placebo arm $(13 \%) .{ }^{16}$

Two trials (CTONG1 104 and WJOG6410L) of adjuvant TKI for EGFR-mutated NSCLC are underway. Both are comparing gefitinib to four cycles of platinum-vinorelbine. PFS is the primary endpoint. ${ }^{17}$

\section{Perioperative antiangiogenic therapy}

There is no biological marker predictive of antiangiogenic drug efficacy but, by analogy with the indication of bevacizumab in advanced NSCLC, most studies of perioperative antiangiogenic therapy have focused on nonsquamous
NSCLC. Several trials of bevacizumab in the adjuvant and neo adjuvant settings are planned or underway. ${ }^{18}$

A single-institution Phase II trial assessed perioperative bevacizumab in patients with operable stage IB-IIIA nonsquamous NSCLC. The primary endpoint was the rate of downstaging among patients with clinical stage IB-IIIA nonsquamous NSCLC treated with neoadjuvant docetaxelcisplatin-bevacizumab combination therapy. Adjuvant bevacizumab $(15 \mathrm{mg} / \mathrm{kg})$ was administered intravenously, starting 42-56 days postoperatively and continuing every 21 days for 1 year (18 cycles). If postoperative radiotherapy was indicated for N2 nodal involvement or positive resection margins, bevacizumab initiation was delayed until 28-52 days after the completion of radiation therapy. No other chemotherapy was given postoperatively. Fifty patients were enrolled between August 2005 and April 2011. The primary endpoint was not met, with downstaging in $38 \%$ of cases. Median OS following the start of neoadjuvant therapy had not been reached, and the 3-year OS was $64 \%$. Downstaging was not associated with improved OS following resection (3-year OS: $70 \%$ vs 56\%, $P=0.24$ ). Nodal downstaging was associated with marginally improved 3 -year OS (66\% vs $37 \%, P=0.051)$.

The US Intergroup Phase III trial, ECOG 1505, will test the following chemotherapy options: cisplatin/vinorelbine, cisplatin/docetaxel, cisplatin/gemcitabine, and cisplatin/ pemetrexed (nonsquamous histology only), with or without bevacizumab, in patients with resected IB-IIIA NSCLC (patients with stage IB disease must have had tumors measuring at least $4 \mathrm{~cm}$ ). The primary endpoint is OS. A total of 1,500 patients will be randomized. Bevacizumab will be started during the first cycle of chemotherapy and continued for up to 1 year. ${ }^{19}$

Another multicenter, randomized, Phase II trial is evaluating the safety and efficacy of carboplatin/docetaxel plus bevacizumab, followed by bevacizumab maintenance therapy, in patients with completely resected Ib/II NSCLC and in selected patients with completely resected stage III NSCLC.

A pharmacogenomic study in patients with completely resected stage IB/IIIA NSCLC who are not fit for cisplatinbased adjuvant chemotherapy and who have excision repair cross-complementing 1 (ERCC1)-positive tumors will evaluate the feasibility of adjuvant therapy with docetaxel plus vinorelbine. ${ }^{20}$ An additional purpose of this study is to establish whether bevacizumab may be safely added to docetaxel plus vinorelbine. Elsewhere, a pilot trial is assessing the safety and feasibility of adding bevacizumab to radiotherapy and cisplatin-based chemotherapy (cisplatin/docetaxel or 
cisplatin/gemcitabine) in resected stage IIIA-N2 NSCLC. Bevacizumab is administered simultaneously with chemotherapy and radiotherapy and is then given as maintenance therapy for 1 year. ${ }^{20}$

\section{Future perspectives: customizing adjuvant therapy}

The literature on the use of biomarkers to guide neoadjuvant therapy in patients with early-stage NSCLC is sparse, and most data come from retrospective analyses of small, uncontrolled studies in which biomarker status was determined only before or after chemotherapy (Table 2). Few studies have evaluated neoadjuvant therapies selected on the basis of molecular tumor analysis. In a Phase II, nonrandomized study, ${ }^{21} 24$ patients with stage IIIA-N2 NSCLC had a molecular analysis: 12 EGFR-mutated patients received 42 days of neoadjuvant erlotinib, and 12 EGFR-wild-type patients received three cycles of cisplatin-gemcitabin. PFS was better in the EGFR-mutated patients but the lack of randomization makes it difficult to conclude.

More data are available in the adjuvant setting. A prospective, randomized, Phase II trial, IFCT-0801 TASTE, allocated adjuvant therapy on the basis of ERCC1 and $E G F R$ mutational status to 150 patients with completely resected nonsquamous stage II or IIIA (non-N2) NSCLC. ${ }^{22}$ Patients in the control arm $(n=74)$ received four courses of standard-dose cisplatin plus pemetrexed. In the customized treatment arm $(\mathrm{n}=76)$, patients with activating EGFR mutations received erlotinib $150 \mathrm{mg}$ for 1 year. ERCC1-negative patients received four courses of cisplatin plus pemetrexed, and ERCC1-positive patients were simply monitored. The aim was to determine the feasibility of customized adjuvant chemotherapy based on timely biomarker analysis (within 2 months postsurgery). Secondary endpoints were tolerability,

Table 2 Customizing adjuvant therapy trials

\begin{tabular}{|c|c|c|c|}
\hline References & Design & $\begin{array}{l}\text { Biomakers } \\
\text { tested }\end{array}$ & Results \\
\hline Wislez et $\mathrm{al}^{22}$ & Phase II, multicentric & ERCCI, EGFR & $\begin{array}{l}\text { ERCCI IHC } \\
\text { unreliable }\end{array}$ \\
\hline Bepler et al ${ }^{19}$ & Phase II, multicentric & ERCCI, RRMI & $\begin{array}{l}\text { ERCCI IHC } \\
\text { unreliable }\end{array}$ \\
\hline Gerber et $\mathrm{a}^{24}$ & Phase III, multicentric & ALK, EGFR & Ongoing \\
\hline Novello et $\mathrm{a}^{25}$ & Phase II, multicentric & $\begin{array}{l}\text { ERCCI, TS } \\
\text { phenotype }\end{array}$ & Ongoing \\
\hline Clinical tria| ${ }^{26}$ & Phase III, multicentric & BRCAI & Ongoing \\
\hline Clinical tria| ${ }^{27}$ & Phase III, multicentric & EGFR & Ongoing \\
\hline
\end{tabular}

Abbreviations: $E R C C I$, excision repair cross-complementing I; RRMI, ribonucleotide reductase MI; TS, thymidylate synthase; EGFR, epidermal growth factor receptor; ALK, anaplastic lymphoma kinase translocation; BRCAI, breast cancer I gene; IHC, immuno-histochemical. compliance with adjuvant therapy, and biomarker distribution. The proportion of patients who had fully documented biomarker status and were able to start adjuvant treatment within 2 months of surgery was $80 \%$, demonstrating the feasibility of a biology-driven trial in the adjuvant NSCLC setting. ${ }^{22}$ However, the Phase III study was canceled because the $E R C C 1$ immunohistochemical readouts were found to be unreliable (Table 2).

A Phase II adjuvant trial (SWOG-0720, NCT00792701) conducted by a cooperative group, using a biomarker-based decision algorithm in patients with stage I NSCLC, was designed to assess the feasibility and preliminary efficacy of assigning patients to therapy or observation by using a molecularly based decision algorithm. ${ }^{23}$ ERCC1 and ribonucleotide reductase M1 (RRMI) were analyzed by immunofluorescence-based in situ automated quantitative image analysis and categorized as high or low using prespecified cutoff values. Patients with high ERCC1 and RRM1 were monitored, and all the other patients were assigned to four cycles of cisplatin plus gemcitabine. Feasibility, defined as treatment assignment within 84 days after surgery in $>85 \%$ of patients, was demonstrated. The 2 -year PFS and OS rates were $80 \%$ and $96 \%$, respectively. RRM1 protein levels fell within the previously established range, while ERCC1 levels were slightly lower than expected. The rates of assignment to observation (22\%) and chemotherapy (78\%) were as expected..$^{23}$ The authors concluded that gene expression analysis for treatment assignment is feasible but that, as in the TASTE study, real-time quantitative in situ ERCC1 and RRM1 analyses require further development. $^{23}$

The Adjuvant Lung Cancer Enrichment Marker Identification and Sequencing trial (ALCHEMIST) is a National Cancer Institute-sponsored initiative designed to address these questions in earlier-stage disease. ${ }^{24} \mathrm{Up}$ to 8,000 patients with pathologically confirmed stage 1-3 nonsquamous NSCLC will be enrolled either before or after surgical resection. Tumors will be centrally genotyped for $E G F R$ mutations and $A L K$ rearrangements. Blood and tumor samples will also be collected for advanced genomic analysis. Patients with EGFR mutations or $A L K$ rearrangements will be randomized to placebo or erlotinib/crizotinib after completion of standard adjuvant therapy. The timing of randomization depends on other therapies: it will take place within 90 days of surgery if no adjuvant therapy is given, within 180 days of surgery if adjuvant chemotherapy is given, and within 270 days of surgery if adjuvant chemotherapy and postoperative radiation therapy are given. The study treatments will be given for up to 2 years, corresponding to the period of greatest risk of relapse 
after surgical resection. Both trials are double-blinded and use OS as the primary endpoint. The ALCHEMIST-EGFR trial will enroll 410 patients, providing $85 \%$ power with a one-sided type I error of 0.05 to demonstrate an OS hazard ratio of 0.67 in favor of erlotinib. The ALCHEMIST-ALK trial will enroll 378 patients, providing $80 \%$ power and a one-sided type I error of 0.05 to demonstrate an OS hazard ratio of 0.67 in favor of crizotinib.

The International Tailored Chemotherapy Adjuvant (ITACA) trial is a Phase III biomarker study using tumoral ERCC1 and thymidylate synthase (TS) messenger RNA expression levels to guide treatment decisions. Patients with pathological stages II and III will be randomized either to a standard cisplatin-based doublet of the investigator's choice or to one of four prespecified regimens determined according to the ERCC1/TS phenotype, as follows: taxane monotherapy for patients with high levels of both biomarkers; single-agent pemetrexed for those with high ERCC1 and low TS expression; cisplatin plus gemcitabine for those with low ERCC1 and high TS expression; and cisplatin plus pemetrexed for those with low levels of both biomarkers. The treatment algorithm is based on the anticipated predictive effects of the two biomarker genes. ${ }^{25}$

The GECP Study of Customized Adjuvant Chemotherapy (SCAT) is a Phase III trial based on a biomarker strategy in which patients with $\mathrm{pN} 1 / \mathrm{N} 2$ disease are randomized to receive either cisplatin plus docetaxel (standard chemotherapy) or to one of three prespecified regimens chosen according to BRCA1 transcript levels: cisplatin plus gemcitabine for patients with low BRCA1 expression; singleagent docetaxel for those with high BRCA1 expression; and cisplatin plus docetaxel for those with intermediate BRCA1 expression. ${ }^{26}$

Another highly anticipated neoadjuvant trial is the Phase II nonrandomized ECON study in which erlotinib is incorporated into a multimodal therapeutic approach: patients with resectable stage IB/IIIA NSCLC and documented EGFR mutations are initially treated with preoperative erlotinib, then cisplatin plus pemetrexed is added before surgical resection, which is itself followed by adjuvant erlotinib. ${ }^{27}$ With the pathological complete response rate as the primary endpoint, the rationale of this study is based on the strikingly high overall response rate achieved with gefitinib in advanced EFGR-mutated disease.

\section{Conclusion}

Customized neo/adjuvant therapy for localized NSCLC, with or without oncogenic driver mutations, remains a major challenge. Large international prospective studies are needed to determine the efficacy of current adjuvant chemotherapy regimens in patients with oncogenic driver mutations, as well as the impact of TKIs and antiangiogenic agents in this setting.

\section{Acknowledgment}

This study was an academic review conducted and funded by Groupe Français de Pneumo Cancerologie (GFPC).

\section{Author contributions}

All authors contributed toward data analysis, drafting and revising the paper and agree to be accountable for all aspects of the work.

\section{Disclosure}

The authors report no conflicts of interest in this work.

\section{References}

1. Kerr KM, Bubendorf L, Edelman MJ, et al. Second ESMO consensus conference on lung cancer: pathology and molecular biomarkers for non-small-cell lung cancer. Ann Oncol. 2014;25:1681-1690.

2. Eberhardt WE, De Ruysscher D, Weder W, et al. 2nd ESMO Consensus Conference in Lung Cancer: locally advanced stage III non-small-cell lung cancer. Ann Oncol. 2015;26:1573-1588.

3. Burdett S, Pignon JP, Tierney J, et al; Non-Small Cell Lung Cancer Collaborative Group. Adjuvant chemotherapy for resected early-stage non-small cell lung cancer. Cochrane Database Syst Rev. 2015;2(3): CD011430.

4. Arriagada R, Dunant A, Pignon JP, et al. Long-term results of the international adjuvant lung cancer trial evaluating adjuvant cisplatin-based chemotherapy in resected lung cancer. J Clin Oncol. 2010;28:35-42.

5. Butts CA, Ding K, Seymour L, et al. Randomized phase III trial of vinorelbine plus cisplatin compared with observation in completely resected stage IB and II non-small-cell lung cancer: updated survival analysis of JBR-10. J Clin Oncol. 2010;28:29-34.

6. Douillard JY, Rosell R, De Lena M, et al. Adjuvant vinorelbine plus cisplatin versus observation in patients with completely resected stage IB-IIIA non small-cell lung cancer (Adjuvant Navelbine International Trialist Association [ANITA]): a randomized controlled trial. Lancet Oncol. 2006;7:719-727.

7. Scagliotti GV, Fossati R, Torri V, et al. Randomized study of adjuvant chemotherapy for completely resected stage I, II, or IIIA non-small-cell lung cancer. J Natl Cancer Inst. 2003;95:1453-1461.

8. Strauss GM, Herndon JE II, Maddaus MA, et al. Adjuvant paclitaxel plus carboplatin compared with observation in stage IB non-small-cell lung cancer: CALGB 9633 with the Cancer and Leukemia Group B, Radiation Therapy Oncology Group, and North Central Cancer Treatment Group Study Groups. J Clin Oncol. 2008;26:5043-5051.

9. NSCLC Meta-analysis Collaborative Group. Preoperative chemotherapy for non-small cell lung cancer: a systematic review and meta-analysis of individual participant data. Lancet. 2014;383:1561-1571.

10. Goss GD, O'Callaghan C, Lorimer I, et al. Gefitinib versus placebo in completely resected non-small-cell lung cancer: results of the NCIC CTG BR19 study. J Clin Oncol. 2013;31:3320-3326.

11. Altorki NK, O'brien MER, Eberhardt WEE, et al. Adjuvant erlotinib (E) versus placebo $(\mathrm{P})$ for completely resected stage IB-IIIA EGFR-positive (IHC/FISH) non-small cell lung cancer (NSCLC): RADIANT Results: Locally Advanced Non-Small Cell Lung Cancer. Int J Rad Oncol Biol Phys. 2014;90:S2-S3.

12. Sun HB, Ou W, Li Y, et al. Epidermal growth factor receptor mutation status and adjuvant chemotherapy in resected advanced non-small-cell lung cancer. Clin Lung Cancer. 2013;14:376-382. 
13. Janjigian YY, Park BJ, Zakowski MF, et al. Impact on disease-free survival of adjuvant erlotinib or gefitinib in patients with resected lung adenocarcinomas that harbor EGFR mutations. J Thorac Oncol. 2011; 6:569-575.

14. Li N, Ou W, Ye X, et al. Pemetrexed-carboplatin adjuvant chemotherapy with or without gefitinib in resected stage IIIA-N2 non-small cell lung cancer harbouring EGFR mutations: a randomized, phase II study. Ann Surg Oncol. 2014;21:2091-2096.

15. Pennell NA. SELECT: A multicenter phase II trial of adjuvant erlotinib in resected early-stage EGFR mutation-positive NSCLC. J Clin Oncol. 2014:Abs 7514

16. Shepperd FA. Adjuvant erlotinib versus placebo in non-small cell lung cancer patients with tumors carrying EGFR-sensitizing mutations from the RADIANT trial. J Clin Oncol. 2014:Abs 7513.

17. Guangdong Association of Clinical Trials. Gefitinib versus vinorelbine/ platinum as adjuvant treatment in stage II-IIIA(N1-N2) NSCLC with EGFR mutation (ADJUVANT). Available from: http://clinicaltrials. gov/ct $2 /$ show $/$ NCT01405079?term $=$ NCT01405079+3\&rank $=1$. NLM identifier: NCT01405079. Accessed June 6, 2016.

18. Schettino C, Bareschino MA, Rossi A, et al. The potential role of bevacizumab in early stages and locally advanced non-small cell lung cancer. Ther Adv Med Oncol. 2009;1:5-13.

19. Bepler G, Zinner RG, Moon J, et al. A phase 2 cooperative group adjuvant trial using a biomarker-based decision algorithm in patients with stage I non-small cell lung cancer (SWOG-0720, NCT00792701). Cancer. 2014;120:2343-2351.

20. Chaft JE, Rusch V, Ginsberg MS, et al. Phase II trial of neoadjuvant bevacizumab plus chemotherapy and adjuvant bevacizumab in patients with resectable nonsquamous non-small-cell lung cancers. J Thorac Oncol. 2013;8:1084-1090.
21. Zhong W. Phase II study of a biomarker-guided neo-adjuvant treatment strategy for IIIA-N2 non-small-cell lung cancer based on EGFR mutation status. J Clin Oncol. 2014:Abs 7572.

22. Wislez M, Barlesi F, Besse B, et al. Customized adjuvant phase II trial in patients with non-small-cell lung cancer: IFCT-0801 TASTE. JClin Oncol. 2014;32:1256-1261.

23. Adjuvant Therapy Based on Gene Expression in Stage IA and IB NonSmall Cell Lung Cancer. Available from: https://clinicaltrials.gov/ct2/ results. Accessed May 28, 2016.

24. Gerber DE, Oxnard GR, Govindan R. ALCHEMIST: Bringing genomic discovery and targeted therapies to early-stage lung cancer. Clin Pharmacol Ther. 2015;97:447-450.

25. Novello S, Manegold C, Grohe C, et al. International tailored chemotherapy adjuvant trial: ITACA trial. J Clin Oncol. 2012;30:abstr TPS7109.

26. Spanish Lung Cancer Group. Randomized customized adjuvant chemotherapy (GECP-SCAT) (GECP-SCAT). Available from: https:/clinicaltrials.gov/ct2/show/NCT00478699 . NLM identifier: NCT00478699. Accessed June 6, 2016.

27. Memorial Sloan Kettering Cancer Center. Erlotinib and chemotherapy for patients with stage IB-IIIA NSCLC with EGFR mutations (ECON). Available from: https://clinicaltrials.gov/ct2/show/NCT00577707. NLM identifier: NCT00577707. Accessed June 6, 2016.
OncoTargets and Therapy

\section{Publish your work in this journal}

OncoTargets and Therapy is an international, peer-reviewed, open access journal focusing on the pathological basis of all cancers, potential targets for therapy and treatment protocols employed to improve the management of cancer patients. The journal also focuses on the impact of management programs and new therapeutic agents and protocols on

\section{Dovepress}

patient perspectives such as quality of life, adherence and satisfaction The manuscript management system is completely online and includes a very quick and fair peer-review system, which is all easy to use. Visit http://www.dovepress.com/testimonials.php to read real quotes from published authors. 